J. Korean Math. Soc. 46 (2009), No. 4, pp. 841-857

DOI 10.4134/JKMS.2009.46.4.841

\title{
ON COMPUTER TOPOLOGICAL FUNCTION SPACE
}

\author{
Sang-Eon Han and Dimitris N. Georgiou
}

\begin{abstract}
In this paper, we give and study the notion of computer topological function space between computer topological spaces with $k_{i}$ adjacency, $i \in\{0,1\}$. Using this notion, we study various properties of topologies of a computer topological function space.
\end{abstract}

\section{Introduction}

In this paper, we consider a set in $\mathbb{Z}^{n}$ as a Khalimsky grid with graph $k$ connectivity, i.e., a Khalimsky topological space with $k$-adjacency. For an introduction and survey to digital and computer topology, see [3, 11, 12, 15, 19, 20]. Recently, the paper [3] investigates various properties of a homeomorphism in Khalimsky topology and the paper [21] establishes a digital version of Jordan curve theorem. Besides, the paper [18] treats an extension problem of a continuous map $f: A \rightarrow \mathbb{Z}$ for $A \subset \mathbb{Z}^{n}$ from a Khalimsky topological point of view. Since a Khalimsky continuous map of two Khalimsky topological spaces need not preserve a $k$-connectivity [12] (see Example 2.4), we need another continuity such as Khalimsky continuity preserving a $k$-connectedness.

In general, by computer or digital topology is meant the mathematical recognition of a set $X \subset \mathbb{Z}^{n}$, e.g., a development of tools implementing topological concepts for use in the sciences and engineering [12]. More precisely, in order to study an object in $\mathbb{Z}^{n}$, while digital topology treats an object in $\mathbb{Z}^{n}$ with both its discrete topological structure and one of the $k$-adjacency relations of $\mathbb{Z}^{n}$, computer topology can uses all kinds of topological structures and related mathematical tools, e.g., Khalimsky product topology with $k$-adjacency and various continuities from the view point of computer topology [12]. Digital and computer topology grew out of digital geometry expanded into applications where significant topological issues arise. Thus, they may be of interest for both computer scientists who try to apply topological knowledge for investigating some properties of an object in $\mathbb{Z}^{n}$ in relation with the studies of image processing, pattern recognition, mathematical morphology, and so forth

Received November 5, 2007.

2000 Mathematics Subject Classification. 54C05, 54C35, 54F05, 68U05.

Key words and phrases. computer topological (product) space, N-compatible, generalized $\left(k_{0}, k_{1}\right)$-continuous function, computer topological function space, $\mathcal{A}$-splitting, $\mathcal{A}$-admissible. 
and mathematicians who want to use a computer to solve complicated topological problems. They play significant roles in computer graphics, image synthesis, image analysis, and so forth [12].

In this paper, we give and study the notion of generalized Khalimsky $\left(k_{0}, k_{1}\right)$ continuous function (briefly, GKD- $\left(k_{0}, k_{1}\right)$-continuous function) between generalized computer topological spaces with $k_{i}$-adjacency, $i \in\{0,1\}$. Using this notion, we study computer topologies on subsets of $\mathbb{Z}^{n}$, where $n$ is a natural number. This study is an application on function spaces.

This paper is organized as follows. In Section 2, we give several basic notions and discuss a limitation of Khalimsky continuity. In Section 3, we define and study the notion of GKD- $\left(k_{0}, k_{1}\right)$-continuity between generalized computer topological spaces with $k_{i}$-adjacency, $i \in\{0,1\}$. In Section 4 , we study some topological properties of a computer topological function space. In Section 5, we give some concluding remarks.

\section{Preliminaries}

By $\mathbb{N}, \mathbb{Z}$, and $\mathbb{R}$ we denote the set of all natural numbers, integers, and real numbers, respectively. Let $a, b \in \mathbb{Z}$ with $a \leq b$ and $[a, b]:=\{x \in \mathbb{R}: a \leq x \leq b\}$. Then, the set

$$
[a, b] \cap \mathbb{Z}
$$

denoted by $[a, b]_{\mathbb{Z}}$ is called a digital interval.

Let $n \in \mathbb{N}, \mathbb{Z}^{n}=\underbrace{\mathbb{Z} \times \cdots \times \mathbb{Z}}_{n \text {-tuples }}$, and $p=\left(p_{1}, \ldots, p_{n}\right), q=\left(q_{1}, \ldots, q_{n}\right) \in$ $\mathbb{Z}^{n}$. We say that two distinct points $p$ and $q$ are $k(m, n)$ - (briefly, $k$-) adjacent according to the number $m[7]$, where $m \in \mathbb{N}$ with $1 \leq m \leq n$, if

(1) there are at most $m$ distinct indices $i$ such that $\left|p_{i}-q_{i}\right|=1$, and

(2) for all indices $i$ such that $\left|p_{i}-q_{i}\right| \neq 1$, we have $p_{i}=q_{i}$.

Hereafter, this operator establishing the adjacency relations of $\mathbb{Z}^{n}$ is called $(C O N \star)$. More precisely, by $N_{k}^{*}(p)$ we denote the set of the points $q \in \mathbb{Z}^{n}$ which are adjacent to a given point $p$ according to $(C O N \star)$ and the number $k:=k(m, n)$ is the cardinal number of $N_{k}^{*}(p)$ called the $k$-neighbors of $p[17]$ expect the point $p$. Consequently, the $k$-adjacency relations of $\mathbb{Z}^{n}$ derived from $(C O N \star)$ are exactly the generalization of the commonly used 4- and 8adjacency of $\mathbb{Z}^{2}$ and 6-, 18-, and 26-adjacency of $\mathbb{Z}^{3}$. Concretely, the number $m$ in $(C O N \star)$ determines one of the $k$-adjacency relations of $\mathbb{Z}^{n}$ [5] (see also $[7,8,10])$, as follows

$$
k \in\left\{3^{n}-1(n \geq 2), 3^{n}-\sum_{t=0}^{r-2} C_{t}^{n} 2^{n-t}-1(2 \leq r \leq n-1, n \geq 3), 2 n(n \geq 1)\right\},
$$

where $C_{t}^{n}=\frac{n !}{(n-t) ! t !}$.

So, for example, we have the following [13].

(i) on $\mathbb{Z}^{2}, k \in\{8,4\}$, 
(ii) on $\mathbb{Z}^{3}, k \in\{26,18,6\}$,

(iii) on $\mathbb{Z}^{4}, k \in\{80,64,32,8\}$,

(iv) on $\mathbb{Z}^{5}, k \in\{242,210,130,50,10\}$, and

(v) on $\mathbb{Z}^{6}, k \in\{728,664,472,232,72,12\}$.

The $k$-adjacency relations of (2.1) can be rewritten in a simpler and more generic form as follows.

Proposition 2.1 ([14]). $k(m, n)=\sum_{i=n-m}^{n-1} 2^{n-i} C_{i}^{n}$, where $C_{i}^{n}=\frac{n !}{(n-i) ! i !}$.

For $n \in \mathbb{N}$, a digital picture is represented as a quadruple $\left(\mathbb{Z}^{n}, k, \bar{k}, X\right)$, where $X$ is a subset of $\mathbb{Z}^{n}$, the number $k$ is for $X$ taken from Proposition 2.1, and $\bar{k}$ is for $\mathbb{Z}^{n}-X$. The pair $(X, k)$ in $\left(\mathbb{Z}^{n}, k, \bar{k}, X\right)$ is called a discrete topological space (or a set) with $k$-adjacency (briefly, a space if not confused). In what follows every space $(X, k)$ is considered in a digital picture $\left(\mathbb{Z}^{n}, k, \bar{k}, X\right)$, where $(k, \bar{k}) \in\{(k, 2 n),(2 n, \bar{k})\}$ with $k \neq \bar{k}$ except for the case $(\mathbb{Z}, 2,2, X)[17]$. But, in this paper we will not concern with the $\bar{k}$-adjacency. For a space $(X, k)$ in $\mathbb{Z}^{n}$ and two points $x$ and $y$ in $X$, if there is a positive integer $m \geq 1$ and a map $f:[0, m]_{\mathbb{Z}} \rightarrow X$ such that $f(0)=x_{0}=x, f(1)=x_{1}, \ldots, f(m)=x_{m}=y$ and further, $x_{i}$ and $x_{i+1}$ are $k$-adjacent, $i \in[0, m-1]_{\mathbb{Z}}$, then the map $f$ is called a $k$-path between $x$ and $y$ in the space $(X, k)$ and the number $m$ is called the length of this $k$-path [17]. If $x=x_{0}=x_{m}=y$, then the above $k$-path $f$ is called closed [17]. If $x \neq y$ and there is a $k$-path between $x$ and $y$, then the points $x$ and $y$ are called $k$-connected. Also, if any two points are $k$-connected, then the space $(X, k)$ is called $k$-connected $[17,20]$. For $(X, k)$, a point $x \in X$ is called isolated if it is not $k$-connected with any point in $X$ [17]. If a set $X$ is a singleton set, then $X$ is assumed to be $k$-connected for any $k$-adjacency. Since a digital object in $\mathbb{Z}^{n}$ can be recognized to be a discrete topological space induced from the discrete topological space $\left(\mathbb{Z}^{n}, T^{n}\right), n \in \mathbb{N}$, we consider the following.

Definition 1 ([7]). Let $(X, k)$ be a (discrete topological) space (or a set) with $k$-adjacency, $x, y \in X$.

(1) By $l_{k}(x, y)$ we denote the length of a shortest simple $k$-path from $x$ to $y$ in $X$. Furthermore, we say that $l_{k}(x, y)=\infty$ if there is no $k$-path from $x$ to $y$.

(2) By $N_{k}(x, \varepsilon)$ we denote the set

$$
\left\{y \in X: l_{k}(x, y) \leq \varepsilon\right\} \cup\{x\} .
$$

Besides, $N_{k}(x, \varepsilon)=\{x\}$ for any $\varepsilon \in \mathbb{N}$ if the point $x$ is isolated. This set is called a $k$-neighborhood of $x$ with radius $\varepsilon$.

Consider a set $X \subset \mathbb{Z}^{n}$ with $k$-adjacency of $\mathbb{Z}^{n}$, denoted by $(X, k)$. In relation to the digital continuity of a map $f:\left(X, k_{0}\right) \rightarrow\left(Y, k_{1}\right)$, we may consider that for two points $x_{0}$ and $x_{1}$ are $k_{0}$-adjacent their images by $f$ are required to be $k_{1}$-adjacent or equal to each other. Thus, motivated by the digital continuity of [20] and the $\left(k_{0}, k_{1}\right)$-continuity of [2], we obtain the notion of digital $\left(k_{0}, k_{1}\right)$-continuity in a form: 
Proposition $2.2([7]$, see also $[9,10])$. Let $\left(X, k_{0}\right)$ and $\left(Y, k_{1}\right)$ be spaces in $\mathbb{Z}^{n_{0}}$ and $\mathbb{Z}^{n_{1}}$, respectively. A function $f: X \rightarrow Y$ is $\left(k_{0}, k_{1}\right)$-continuous if and only if for every $x_{0} \in X, \varepsilon \in \mathbb{N}$, and $N_{k_{1}}\left(f\left(x_{0}\right), \varepsilon\right) \subset Y$, there is $\delta \in \mathbb{N}$ such that the corresponding $N_{k_{0}}\left(x_{0}, \delta\right) \subset X$ satisfies $f\left(N_{k_{0}}\left(x_{0}, \delta\right)\right) \subset N_{k_{1}}\left(f\left(x_{0}\right), \varepsilon\right)$.

The current $\left(k_{0}, k_{1}\right)$-continuity is a generalization of the digital continuities of $[2,20]$ without any limitation of the $k$-adjaceny relations of $\mathbb{Z}^{n}$ and has been often used in studying digital covering theory [7, 11, 13], digital $k$-curve, and digital $k$-surface theory $[10,14]$. Furthermore, this digital continuity has the almost pasting property [16].

By the use of the $\left(k_{0}, k_{1}\right)$-continuity of Proposition 2.2, we obtain the digital topological category [11], briefly DTC, consisting of two things:

(1) A class of objects $(X, k)$ in $\mathbb{Z}^{n}$;

(2) For every ordered pair of objects $\left(X, k_{0}\right)$ in $\mathbb{Z}^{n_{0}}$ and $\left(Y, k_{1}\right)$ in $\mathbb{Z}^{n_{1}}$, all $\left(k_{0}, k_{1}\right)$-continuous maps $f:\left(X, k_{0}\right) \rightarrow\left(Y, k_{1}\right)$ as morphisms.

Theorem 2.3 ([6], see also [13]). Let $\left(X, k_{0}\right)$ and $\left(Y, k_{1}\right)$ be spaces in $\mathbb{Z}^{n_{0}}$ and $\mathbb{Z}^{n_{1}}$, respectively. For a $\left(k_{0}, k_{1}\right)$-continuous function $f: X \rightarrow Y$ of Proposition 2.2, we may take $\varepsilon=1=\delta$.

For a $k$-adjacency relation of $\mathbb{Z}^{n}$, we recall that a simple closed $k$-curve with $l$ elements in $X \subset \mathbb{Z}^{n}$ is the image of a $(2, k)$-continuous function $f:[0, l-1]_{\mathbb{Z}} \rightarrow$ $X$ such that $f(i)$ and $f(j)$ are $k$-adjacent if and only if either $j=i+1(\bmod l)$ or $i=j+1(\bmod l)[17]$. Thus, we may use the notation $S C_{k}^{n, l}$ which can be assumed to be a sequence $\left(c_{i}\right)_{i \in[0, l-1]_{\mathbb{Z}}}$ with $f(i)=c_{i}[7]$.

A space $(X, k)$ in $\mathbb{Z}^{n}$ can be considered as a digital graph $G_{k}$ [8] (see also $[6,13])$. To be specific, the vertex set of $G_{k}$ can be recognized to be the set of points of $X$. Besides, two points $x_{1}, x_{2} \in X$ determine a $k$-edge of $G_{k}$ if and only if $x_{1}$ and $x_{2}$ are $k$-adjacent in $X$ [8] (see also [10, 13]). Thus, digital graph versions of the $\left(k_{0}, k_{1}\right)$-continuity, the $\left(k_{0}, k_{1}\right)$-homeomorphism, the $\left(k_{0}, k_{1}\right)$ covering, and the $\left(k_{0}, k_{1}\right)$-homotopy in digital topology were established in [8].

Definition 2 ([2], see also $[8,10,13])$. For two spaces $\left(X, k_{0}\right)$ in $\mathbb{Z}^{n_{0}}$ and $\left(Y, k_{1}\right)$ in $\mathbb{Z}^{n_{1}}$, a map $h: X \rightarrow Y$ is called a $\left(k_{0}, k_{1}\right)$-isomorphism if $h$ is a $\left(k_{0}, k_{1}\right)$-continuous bijection and further, $h^{-1}: Y \rightarrow X$ is $\left(k_{1}, k_{0}\right)$-continuous. Then, we use the notation $X \approx_{\left(k_{0}, k_{1}\right)} Y$. If $n_{0}=n_{1}$ and $k_{0}=k_{1}$, then we call it a $k_{0}$-isomorphism and use the notation $X \approx_{k_{0}} Y$.

Khalimsky line topology on $\mathbb{Z}[3,18]$ is induced from the subbasis

$$
\left\{[2 n-1,2 n+1]_{\mathbb{Z}}: n \in \mathbb{Z}\right\} \text {. }
$$

The family

$$
\{\{2 n+1\}: n \in \mathbb{Z}\} \cup\left\{[2 n-1,2 n+1]_{\mathbb{Z}}: n \in \mathbb{Z}\right\}
$$

is a basis for Khalimsky line topology on $\mathbb{Z}$. We denote this topology $T$. It follows easily that an even point is closed and an odd point is open. Indeed, we have that the smallest open neighborhood is $\{n\}$ if $n$ is odd and $\{n-1, n, n+1\}$ 
if $n$ is even $[3,18,21]$. Now, we consider the product topology on $\mathbb{Z}^{n}$, denoted by $T^{n}$, derived from the Khalimsky line topology on $\mathbb{Z}$.

The topology $T^{n}$ on $\mathbb{Z}^{n}$ is called the Khalimsky topology on $\mathbb{Z}^{n}, n \geq 2$, and the corresponding topological space is denoted by $\left(\mathbb{Z}^{n}, T^{n}\right)$. Also, by some authors, this space is called the Khalimsky $n$-space. In this space, points with all coordinates even are closed and points with all coordinates odd are open $[3,15]$. The other points in $\mathbb{Z}^{n}$ are called mixed points. In Figures 1, 2, 3, and 4 , the symbols $\mathbf{\square}$ and mean a pure closed point and a mixed point, respectively. Besides, a jumbo dot stands for a pure open point.

Definition 3 ([12]). Let $X$ be a set in $\mathbb{Z}^{n}$. We consider the subspace induced from $\left(\mathbb{Z}^{n}, T^{n}\right)$ and denote the corresponding topological space as $\left(X, T_{X}^{n}\right)$. Furthermore, considering the topological space $\left(X, T_{X}^{n}\right)$ with $k$-adjacency, we call it a computer topological space with $k$-adjacency and we briefly use the notation $\left(X, k, T_{X}^{n}\right):=X_{n, k}$.

For two spaces $\left(X, k_{0}\right)$ and $\left(Y, k_{1}\right)$, and a map $f:\left(X, k_{0}\right) \rightarrow\left(Y, k_{1}\right)$, the preservation of the $k_{0}$-connectivity into the $k_{1}$-connectivity is helpful to study digital topology. Meanwhile, we observe that Khalimsky continuity of two Khalimsky topological spaces $X_{n_{0}, k_{0}}$ and $Y_{n_{1}, k_{1}}$, denoted by $f: X_{n_{0}, k_{0}} \rightarrow$ $Y_{n_{1}, k_{1}}$, need not satisfy the requirement [12] (see Example 2.4).

Example 2.4 (Limitation of Khalimsky continuity). Consider a map $f$ : $X_{2,8} \rightarrow Y_{1,2}$ in Figure 1 . Now, let $X=\left\{x_{0}=(2,1), x_{1}=(1,2), x_{2}=\right.$ $\left.(0,3), x_{3}=(1,4), x_{4}=(2,4), x_{5}=(2,5)\right\}$ and $Y=\{1,2,3\}$. We consider the map $f: X_{2,8} \rightarrow Y_{1,2}$ for which $f\left(\left\{x_{1}, x_{2}\right\}\right)=\{1\}, f\left(x_{0}\right)=3$, and $f\left(\left\{x_{3}, x_{4}, x_{5}\right\}\right)=\{2\}$. While $f$ is a Khalimsky continuous map, $f$ cannot preserve the 8 -connectivity into the 2 -connectivity (see the point $x_{0}$ ).

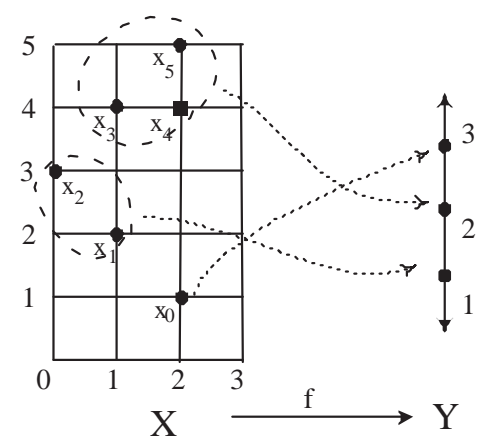

Figure 1. Non-preservation of the 8-connectivity of Khalimsky continuity

In view of Example 2.4, we need to establish a new continuity for the study of Khalimsky spaces. As referred above, since a Khalimsky continuous map $f$ : 
$X_{n_{0}, k_{0}} \rightarrow Y_{n_{1}, k_{1}}$ need not preserve the $k_{0}$-connectivity into the $k_{1}$-connectivity, we need to establish another continuity which is Khalimsky continuous and preserves the $k_{0}$-connectivity into the $k_{1}$-connectivity, as follows.

Definition 4 ([12]). Let $X_{n_{0}, k_{0}}$ and $Y_{n_{1}, k_{1}}$ be two computer topological spaces with $k_{i}$-adjacency, $i \in\{0,1\}$, and $x \in X$. A map $f: X \rightarrow Y$ is called Khalimsky $\left(k_{0}, k_{1}\right)$-continuous (briefly, $\mathrm{KD}-\left(k_{0}, k_{1}\right)$-continuous) at a point $x$ if

(1) $f$ is Khalimsky (topologically) continuous at the point $x$ and

(2) for $N_{k_{1}}(f(x), 1) \subset Y$, there is $N_{k_{0}}(x, 1) \subset X$ such that $f\left(N_{k_{0}}(x, 1)\right) \subset$ $N_{k_{1}}(f(x), 1)$.

Also, a map $f: X \rightarrow Y$ is called $K D-\left(k_{0}, k_{1}\right)$-continuous if the map $f$ is $\mathrm{KD}-\left(k_{0}, k_{1}\right)$-continuous at every point $x \in X$. In addition, if $n_{0}=n_{1}$ and $k_{0}=k_{1}$, then $f: X \rightarrow Y$ is called $K D$ - $k_{0}$-continuous.

We can see that none of the conditions (1) and (2) of Definition 4 implies the other [12], as follows.

Remark 2.5. We now show that the condition (2) of Definition 4 does not hold the condition (1). Let $X=\{(1,1),(1,2),(1,3),(2,2),(2,3),(3,3),(3,1),(3,2)\}$ and $Y=\{0,1,2\}$. We consider a map $f: X_{2,4} \rightarrow Y_{1,2}$ (see Figure 2) for which $f(\{(1,1),(1,2)\})=\{0\}, f(\{(1,3),(2,3),(3,3),(2,2)\})=\{1\}$, and $f(\{(3,2),(3,1)\})=\{2\}$. While the map $f$ satisfies the condition (2) of Definition 4 at the point $(2,2), f$ cannot be Khalimsky continuous at the point $(2,2)$. To be specific, for an open set $\{1\}$ of 1 , since the smallest open set containing the pure closed point $(2,2)$ is the total set $X \in T_{X}^{2}$, we observe $f(X) \nsubseteq\{1\}$.

Next, consider the map $f$ in Example 2.4 (see Figure 1). While the map $f$ satisfies the condition (1) of Definition $4, f$ cannot satisfy the condition (2) at the points $x_{0}$ and $x_{1}$ of $X$ (see Figure 1 ).

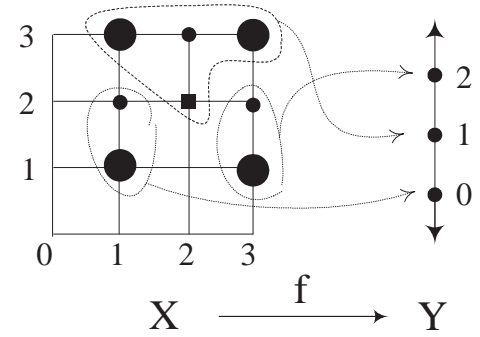

Figure 2. Non-existence of the Khalimsky continuity

We now have a new topological category so called a KD-computer topological category, briefly $K D T C[12]$, consisting of three things:

(1) A class of objects $X_{n, k}$; 
(2) For every ordered pair of objects $X_{n_{0}, k_{0}}$ and $Y_{n_{1}, k_{1}}$ as morphisms, all KD- $\left(k_{0}, k_{1}\right)$-continuous maps $f: X_{n_{0}, k_{0}} \rightarrow Y_{n_{1}, k_{1}}$;

(3) For every ordered triple of objects $X_{n_{0}, k_{0}}, Y_{n_{1}, k_{1}}$, and $Z_{n_{2}, k_{2}}$ and a function associating to a pair of morphisms $f: X_{n_{0}, k_{0}} \rightarrow Y_{n_{1}, k_{1}}$ which is a $\mathrm{KD}-\left(k_{0}, k_{1}\right)$-continuous map and $g: Y_{n_{1}, k_{1}} \rightarrow Z_{n_{2}, k_{2}}$ which is a $\mathrm{KD}-\left(k_{1}, k_{2}\right)$ continuous map, their composite $g \circ f: X_{n_{0}, k_{0}} \rightarrow Z_{n_{2}, k_{2}}$ which is a KD- $\left(k_{0}, k_{2}\right)$ continuous map.

\section{On GKD- $\left(k_{0}, k_{1}\right)$-continuous functions}

In general, on the set $\mathbb{Z}$ we can consider many topologies. For instance, we can consider the Scott topology and the upper topology on $\mathbb{Z}[4]$.

Example 3.1. (1) Let $z \in \mathbb{Z}$ and $\downarrow z=\{x \in \mathbb{Z}, x \leq z\}$. The family consisting of $\mathbb{Z}$ and the set $\{\mathbb{Z}-\downarrow z, z \in \mathbb{Z}\}$ defines a topology on $\mathbb{Z}$, denoted here by $\tau_{u p}$, which coincides with the Scott topology on $\mathbb{Z}$.

Furthermore, we can consider the product topology $\tau_{u p}^{n}$ on $\mathbb{Z}^{n}$ derived from the topology $\tau_{u p}$.

(2) Let $z \in \mathbb{Z}$ and $\uparrow z=\{x \in \mathbb{Z}, z \leq x\}$. The family consisting of $\mathbb{Z}$ and the set $\{\mathbb{Z}-\uparrow z, z \in \mathbb{Z}\}$ defines a topology on $\mathbb{Z}$, denoted by $\tau_{l o}$, which is called lower topology.

Also, we can consider the product topology $\tau_{l o}^{n}$ on $\mathbb{Z}^{n}$ derived from the topology $\tau_{l o}$.

As a generalization of the computer topological space in Definition 3, we obtain the following.

Definition 5. Let $\tau$ be an arbitrary topology on $\mathbb{Z}$ and let $(X, k)$ be a space in $\mathbb{Z}^{n}$. We consider the subspace induced from $\left(\mathbb{Z}^{n}, \tau^{n}\right)$, denoted by $\left(X, \tau_{X}^{n}\right)$. Furthermore, considering the topological space $\left(X, \tau_{X}^{n}\right)$ with $k$-adjacency, we call it a generalized computer topological space with $k$-adjacency and we use the notation $\left(X, k, \tau_{X}^{n}\right)$.

If $\tau$ is the Khalimsky line topology $T$, then the notion of generalized computer topological space with $k$-adjacency coincides with that of computer topological space with $k$-adjacency.

Besides, the space $\left(X, k, \tau_{X}^{n}\right)$ in Definition 5 will be often called briefly a generalized computer topological space if not confused.

Definition 6. Let $\left(X, k_{0}, \tau_{X}^{n_{0}}\right)$ and $\left(Y, k_{1}, \tau_{Y}^{n_{1}}\right)$ be two generalized computer topological spaces and $x_{0} \in X$. A function $f: X \rightarrow Y$ is called GKD- $\left(k_{0}, k_{1}\right)$ continuous at the point $x_{0}$ if

(1) $f$ is topologically continuous at the point $x_{0}$ and

(2) for any $N_{k_{1}}\left(f\left(x_{0}\right), 1\right) \subset Y$, there is $N_{k_{0}}\left(x_{0}, 1\right) \subset X$ such that

$$
f\left(N_{k_{0}}\left(x_{0}, 1\right)\right) \subset N_{k_{1}}\left(f\left(x_{0}\right), 1\right) .
$$


Also, we say that the map $f: X \rightarrow Y$ is $G K D-\left(k_{0}, k_{1}\right)$-continuous if the map $f$ is GKD- $\left(k_{0}, k_{1}\right)$-continuous at every point $x \in X$. In addition, if $n_{0}=n_{1}$ and $k_{0}=k_{1}$, then $f: X \rightarrow Y$ is called $G K D$ - $k_{0}$-continuous.

If $\tau$ is the Khalimsky line topology $T$, then the notion of GKD- $\left(k_{0}, k_{1}\right)$ continuity coincides with the notion of $\mathrm{KD}-\left(k_{0}, k_{1}\right)$-continuity.

Example 3.2. Consider two spaces $X:=\left\{x_{i}\right\}_{i \in[1,10]_{\mathbb{Z}}}$ with $X_{2,8}$ and $Y:=$ $\left\{y_{j}\right\}_{j \in[1,6]_{\mathbb{Z}}}$ with $Y_{2,8}$ (see Figure 3 ). Then consider the map $f: X \rightarrow Y$ for which

$f\left(x_{1}\right)=y_{1}, f\left(\left\{x_{2}, x_{3}, x_{4}\right\}\right)=\left\{y_{2}\right\}, f\left(\left\{x_{5}, x_{6}\right\}\right)=\left\{y_{3}\right\}, f\left(x_{7}\right)=y_{4}, f\left(x_{8}\right)=y_{5}$, and $f\left(\left\{x_{9}, x_{10}\right\}\right)=\left\{y_{6}\right\}$.

Then the map $f$ is a GKD-8-continuous map.

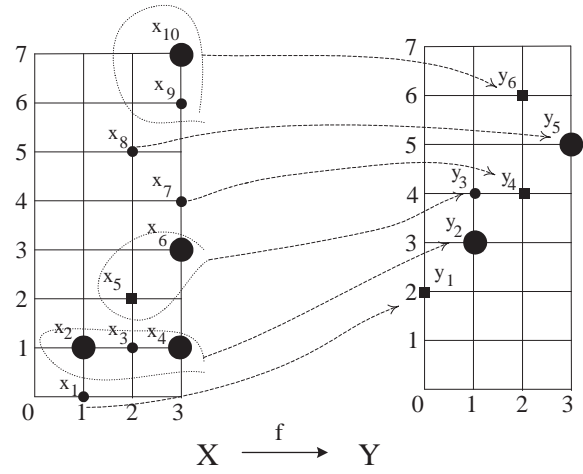

Figure 3. GKD- $(8,8)$-continuity

Theorem 3.3. Let $\left(X, k_{0}, \tau_{X}^{n_{1}}\right)$, $\left(Y, k_{1}, \tau_{Y}^{n_{2}}\right)$, and $\left(Z, k_{2}, \tau_{Z}^{n_{3}}\right)$ be three generalized computer topological spaces. If the map $f_{1}: X \rightarrow Y$ is $G K D-\left(k_{0}, k_{1}\right)$ continuous and the map $f_{2}: Y \rightarrow Z$ is $G K D-\left(k_{1}, k_{2}\right)$-continuous, then the composition $f_{2} \circ f_{1}: X \rightarrow Z$ is GKD- $\left(k_{0}, k_{2}\right)$-continuous.

Proof. First, the map $f_{2} \circ f_{1}$ is obviously continuous.

Second, it suffices to prove that for every $x \in X$ and for every $N_{k_{2}}\left(\left(f_{2} \circ\right.\right.$ $\left.\left.f_{1}\right)(x), 1\right)$, there exists $N_{k_{0}}(x, 1)$ such that

$$
\left(f_{2} \circ f_{1}\right)\left(N_{k_{0}}(x, 1)\right) \subset N_{k_{2}}\left(\left(f_{2} \circ f_{1}\right)(x), 1\right) .
$$

Since the map $f_{2}: Y \rightarrow Z$ is $\operatorname{GKD}-\left(k_{1}, k_{2}\right)$-continuous for any $y \in Y$, we have

$$
f_{2}\left(N_{k_{1}}(y, 1)\right) \subset N_{k_{2}}\left(\left(f_{2}(y), 1\right) .\right.
$$

Also, since the map $f_{1}: X \rightarrow Y$ is GKD- $\left(k_{0}, k_{1}\right)$-continuous, we obtain

$$
f_{1}\left(N_{k_{0}}(x, 1)\right) \subset N_{k_{1}}\left(f_{1}(x), 1\right) .
$$


Thus,

$$
\left(f_{2} \circ f_{1}\right)\left(N_{k_{0}}(x, 1)\right) \subset f_{2}\left(N_{k_{1}}\left(f_{1}(x), 1\right)\right) \subseteq N_{k_{2}}\left(\left(f_{2} \circ f_{1}\right)(x), 1\right),
$$

which completes the proof.

We obviously obtain the following.

Theorem 3.4. Let $\left(X, k, \tau_{X}^{n}\right)$ be a generalized computer topological space. Then, the map id $: X \rightarrow X$ for which $i d(x)=x$ is $G K D-(k, k)$-continuous.

Let us now study a normally compatible $k$-adjacency of a Cartesian product (or a digital product). For two spaces $\left(X, k_{1}\right)$ in $\mathbb{Z}^{n_{1}},\left(Y, k_{2}\right)$ in $\mathbb{Z}^{n_{2}}$, the product space $X \times Y$ in $\mathbb{Z}^{n_{1}+n_{2}}$ can be considered with an N-compatible $k$-adjacency of $\mathbb{Z}^{n_{1}+n_{2}}$ related to the $k_{1}$-and $k_{2}$-adjacencies. Indeed, the establishment of the N-compatible adjacency plays an important role in studying a computer topological product space in relation with the study of a computer topological function space.

Definition 7. For two spaces $\left(X, k_{1}\right)$ in $\mathbb{Z}^{n_{1}},\left(Y, k_{2}\right)$ in $\mathbb{Z}^{n_{2}}$, consider the digital product $X \times Y:=\{(x, y) \mid x \in X, y \in Y\} \subset \mathbb{Z}^{n_{1}+n_{2}}$. Then a $k$-adjacency of two points $(x, y) \in X \times Y,\left(x^{\prime}, y^{\prime}\right) \in X \times Y$ is called normally compatible (briefly, N-compatible) with the $k_{i}$-adjacency, $i \in\{1,2\}$, if

(1) whenever $y=y^{\prime}$ and $(x, y)$ is $k$-adjacent to $\left(x^{\prime}, y^{\prime}\right)$, then $x$ is $k_{1}$-adjacent to $x^{\prime}$;

(2) whenever $x=x^{\prime}$ and $(x, y)$ is $k$-adjacent to $\left(x^{\prime}, y^{\prime}\right)$, then $y$ is $k_{2}$-adjacent to $y^{\prime}$; and

(3) whenever $x \neq x^{\prime}$ and $y \neq y^{\prime}$, and $(x, y)$ is $k$-adjacent to $\left(x^{\prime}, y^{\prime}\right)$, then $x$ is $k_{1}$-adjacent to $x^{\prime}$ and $y$ is $k_{2}$-adjacent to $y^{\prime}$.

The current $\mathrm{N}$-compatible $k$-adjacency is different from the adjacency of the normal product in [1]. The following simple closed 4 - and 8-curves in $\mathbb{Z}^{2}$ in $[6,9,13]$ and a simple closed 18 - and 26 -curves in $\mathbb{Z}^{3}$ will be often used later in this paper [14].

$$
\left\{\begin{array}{c}
S C_{4}^{2,8} \approx_{4}((0,0),(0,1),(0,2),(1,2),(2,2),(2,1),(2,0),(1,0)), \\
S C_{8}^{2,4}:=M S C_{8}^{\prime} \approx_{8}((0,0),(1,1),(2,0),(1,-1)), \\
S C_{8}^{2,6}:=M S C_{8} \approx_{8}((0,0),(1,1),(1,2),(0,3),(-1,2),(-1,1)), \\
S C_{8}^{2,8}:=((0,0),(1,1),(2,2),(1,3),(0,4),(-1,3),(-2,2),(-1,1)), \\
M S C_{18}:=((0,0,0),(1,-1,0),(1,-1,1),(2,0,1),(1,1,1),(1,1,0)), \\
S C_{18}^{3,6}:=((0,0,0),(1,0,1),(1,1,2),(0,2,2),(-1,1,2),(-1,0,1))
\end{array}\right\}
$$

Example 3.5. For two spaces $(X, 2)$ and $(Y, 2)$ in $\mathbb{Z}$, the Cartesian product $X \times Y$ can be assumed on $\mathbb{Z}^{2}$ with an N-compatible 8 -adjacency. Besides, we obtain the following.
(a) $\left(S C_{8}^{2,6} \times[a, b]_{\mathbb{Z}}, 26\right)$, 


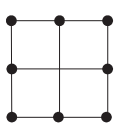

$\mathrm{SC}_{4}^{2,8}$

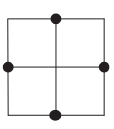

$\mathrm{SC}_{8}^{2,4}$

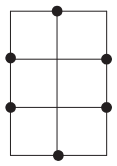

$\mathrm{SC}_{8}^{2,6}$

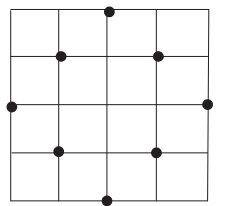

$\mathrm{SC}_{8}^{2,8}$

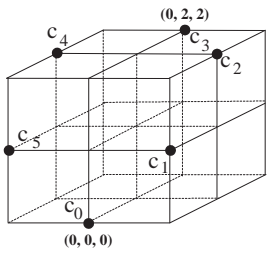

$\mathrm{SC}_{18}^{3,6}$

FIGURE 4. Various simple closed $k$-curves in $[6,9,13,14]$

(b) $\left(S C_{8}^{2,6} \times S C_{8}^{2,6}, 80\right)$,

(c) $\left(S C_{18}^{3,6} \times S C_{8}^{2,4}, k\right), k \in\{210,242\}$, and

(d) No N-compatible $k$-adjacency of $S C_{4}^{2,8} \times S C_{8}^{2,6}$ exists.

Proof. (a), (b), and (c) are obviously proved.

(d) Let us examine some N-compatible $k$-adjacency of $S C_{4}^{2,8} \times S C_{8}^{2,6} \subset \mathbb{Z}^{4}$. First, suppose an N-compatible $k$-adjacency of $S C_{4}^{2,8} \times S C_{8}^{2,6}$. By Definition 7(3), we can assume an N-compatible $k$-adjacency of $S C_{4}^{2,8} \times S C_{8}^{2,6}, k \in$ $\{64,80\}$. Then the $k$-adjacency contradicts to (1) and (2) of Definition 7 .

Second, if we assume the $k$-adjacency of $S C_{4}^{2,8} \times S C_{8}^{2,6}, k \in\{8,32\}$, then it cannot satisfy Definition 7(3).

In view of Example 3.5, we observe that for a space $\left(X_{i}, k_{i}\right)$ the existence of an N-compatible $k$-adjacency of the digital product $X_{1} \times X_{2}$ depends on the situation. Furthermore, the N-compatible $k$-adjacency of Definition 7 obviously implies the following.

Theorem 3.6. Let $\left(X, k_{1}, \tau_{X}^{n_{1}}\right)$ and $\left(Y, k_{2}, \tau_{Y}^{n_{2}}\right)$ be two generalized computer topological spaces such that the product spaces $\left(X \times Y, k, \tau_{X \times Y}^{n_{1}+n_{2}}\right)$ is well defined with $N$-compatible $k$-adjacency. Then, each projection map $p_{i}: X \times Y \rightarrow X$ or $Y$ is a $G K D-\left(k, k_{i}\right)$-continuous map, $i \in\{1,2\}$.

Theorem 3.7. Let $\left(X, k_{1}, \tau_{X}^{n_{1}}\right),\left(Y, k_{2}, \tau_{Y}^{n_{2}}\right)$, and $\left(Z, k_{3}, \tau_{Z}^{n_{3}}\right)$ be three generalized computer topological spaces. Assume that the product spaces $(X \times$ $\left.Z, k, \tau_{X \times Z}^{n_{1}+n_{3}}\right)$ and $\left(Y \times Z, k^{\prime}, \tau_{Y \times Z}^{n_{2}+n_{3}}\right)$ are well defined with $N$-compatible $k$ - 
and $k^{\prime}$-adjacencies, respectively. If the map $f: X \rightarrow Y$ is a GKD- $\left(k_{1}, k_{2}\right)$ continuous map, then the map $f \times i d: X \times Z \rightarrow Y \times Z$ is $G K D-\left(k, k^{\prime}\right)$ continuous, where id $: Z \rightarrow Z$ is the identity map.

Proof. Obviously, the map $f \times i d$ is continuous.

Now, for $(x, z) \in X \times Z$, let

$$
N_{k^{\prime}}((f \times i d)(x, z), 1)=N_{k^{\prime}}((f(x), z), 1) \subseteq Y \times Z .
$$

Let us now show

$$
(f \times i d)\left(N_{k}((x, z), 1)\right) \subseteq N_{k^{\prime}}((f(x), z), 1) .
$$

Indeed, since the map $f: X \rightarrow Y$ is GKD- $\left(k_{1}, k_{2}\right)$-continuous for $N_{k_{1}}(f(x), 1)$ $\subseteq Y$, there exists $N_{k_{1}}(x, 1) \subseteq X$ such that

$$
f\left(N_{k_{1}}(x, 1)\right) \subseteq N_{k_{2}}(f(x), 1) .
$$

Also, since the map $i d: Z \rightarrow Z$ is GKD- $k_{3}$-continuous for $N_{k_{3}}(z, 1) \subseteq Z$, we obtain:

$$
i d\left(N_{k_{3}}(z, 1)\right) \subseteq N_{k_{3}}(z, 1) .
$$

Let us now prove that the relation (3.1) is true. Let $\left(x^{\prime}, z^{\prime}\right) \in N_{k}((x, z), 1)$. Then, by Definition 7 , we have that $x^{\prime} \in N_{k_{1}}(x, 1)$ and $z^{\prime} \in N_{k_{3}}(z, 1)$. Thus we have:

and

$$
f\left(x^{\prime}\right) \in N_{k_{2}}(f(x), 1)
$$

Therefore, we have:

$$
i d\left(z^{\prime}\right)=z^{\prime} \in N_{k_{3}}(z, 1)
$$

$$
(f \times i d)\left(x^{\prime}, z^{\prime}\right)=\left(f\left(x^{\prime}\right), z^{\prime}\right) \in N_{k^{\prime}}((f(x), z), 1),
$$

which completes the proof.

Theorem 3.8. Let $\left(X, k_{1}, \tau_{X}^{n_{1}}\right),\left(Y, k_{2}, \tau_{Y}^{n_{2}}\right)$, and $\left(Z, k_{3}, \tau_{Z}^{n_{3}}\right)$ be three generalized computer topological spaces. Assume that the product space $(X \times$ $\left.Y, k, \tau_{X \times Y}^{n_{1}+n_{2}}\right)$ is well defined with an $N$-compatible $k$-adjacency. If a map $F$ : $X \times Y \rightarrow Z$ is $G K D-\left(k, k_{3}\right)$-continuous and $x \in X$, then the map $F_{x}: Y \rightarrow Z$ for which $F_{x}(y)=F(x, y)$ for every $y \in Y$ is $G K D-\left(k_{2}, k_{3}\right)$-continuous.

Proof. Obviously, the map $F_{x}: Y \rightarrow Z$ is continuous.

Now, let $y \in Y$ and let $N_{k_{3}}\left(F_{x}(y), 1\right) \subseteq Z$. Then we prove

$$
F_{x}\left(N_{k_{2}}(y, 1)\right) \subseteq N_{k_{3}}\left(F_{x}(y), 1\right) .
$$

Indeed, since the map $F: X \times Y \rightarrow Z$ is GKD- $\left(k, k_{3}\right)$-continuous and $F_{x}(y)=$ $F(x, y)$ for $N_{k_{3}}(F(x, y), 1) \subseteq Z$, there exists $N_{k}((x, y), 1) \subseteq X \times Y$ such that

$$
F\left(N_{k}((x, y), 1)\right) \subseteq N_{k_{3}}(F(x, y), 1) .
$$

We suffice to prove that

$$
F_{x}\left(N_{k_{2}}(y, 1)\right) \subset N_{k_{3}}\left(F_{x}(y), 1\right) .
$$


Indeed, let $y^{\prime} \in N_{k_{2}}(y, 1)$, then by Definition 7 we have $\left(x, y^{\prime}\right) \in N_{k}((x, y), 1)$ and, therefore,

which completes the proof.

$$
F_{x}\left(y^{\prime}\right)=F\left(x, y^{\prime}\right) \in N_{k_{3}}(F(x, y), 1),
$$

\section{Topologies on the GKD- $\left(k_{0}, k_{1}\right)$-continuous function space}

In order to investigate some topologies of a computer topological function space, we need the following.

Notation 1. Let $\left(Y, k_{2}, \tau_{Y}^{n_{2}}\right)$ and $\left(Z, k_{3}, \tau_{Z}^{n_{3}}\right)$ be two generalized computer topological spaces. By $C(Y, Z)$ we denote the set of all GKD- $\left(k_{2}, k_{3}\right)$-continuous functions from $Y$ into $Z$. Let $f \in C(Y, Z)$. We can assume that

$f=\left\{\left(\left(p_{1}, \ldots, p_{n_{2}}\right),\left(q_{1}, \ldots, q_{n_{3}}\right)\right): f\left(p_{1}, \ldots, p_{n_{2}}\right)=\left(q_{1}, \ldots, q_{n_{3}}\right),\left(p_{1}, \ldots, p_{n_{2}}\right) \in Y\right\}$.

By the relation (4.1), if we assume

$$
\left(\left(p_{1}, \ldots, p_{n_{2}}\right),\left(q_{1}, \ldots, q_{n_{3}}\right)\right) \equiv\left(p_{1}, \ldots, p_{n_{2}}, q_{1}, \ldots, q_{n_{3}}\right),
$$

then we can consider each map $f \in C(Y, Z)$ as a subset of $\mathbb{Z}^{n_{2}+n_{3}}$.

In what follows by

$$
C\langle Y, Z\rangle
$$

we denote the set

$$
\bigcup\{f: f \in C(Y, Z)\} \text {. }
$$

Clearly, $C\langle Y, Z\rangle \subseteq \mathbb{Z}^{n_{2}+n_{3}}$. Also, we consider the space $\left(C\langle Y, Z\rangle, k^{\prime}\right)$, where $k^{\prime}:=k^{\prime}(m, n)=\sum_{i=n-m}^{n-1} 2^{n-i} C_{i}^{n}$ in Proposition 2.1 and $n=n_{2}+n_{3}$.

Example 4.1. Assume that $Y=\{1\} \subseteq \mathbb{Z}$ and $Z=\{3,4\} \subseteq \mathbb{Z}$. We consider the spaces $(Y, 2)$ and $(Z, 2)$ with the Khalimsky topologies $T_{Y}$ and $T_{Z}$, respectively. In this case, $C(Y, Z)=\{f, g\}$, where $f=\{(1,3)\}$ and $g=\{(1,4)\}$. Clearly, $C\langle Y, Z\rangle=\{(1,3),(1,4)\} \subseteq \mathbb{Z}^{2}$. So, we can consider the computer topological spaces $\left(C\langle Y, Z\rangle, 4, T_{C\langle Y, Z\rangle}^{2}\right)$ and $\left(C\langle Y, Z\rangle, 8, T_{C\langle Y, Z\rangle}^{2}\right)$.

Notation 2. Let $\left(X, k_{1}, \tau_{X}^{n_{1}}\right),\left(Y, k_{2}, \tau_{Y}^{n_{2}}\right)$, and $\left(Z, k_{3}, \tau_{Z}^{n_{3}}\right)$ be three generalized computer topological spaces such that the product $X \times Y$ is well defined with an N-compatible $k$-adjacency and let $F: X \times Y \rightarrow Z$ be a GKD- $\left(k, k_{3}\right)$-continuous map.

For every $x \in X$, by $F_{x}$ we denote the GKD- $\left(k_{2}, k_{3}\right)$-continuous map (see Theorem 3.8) from $Y$ into $Z$, for which $F_{x}(y)=F(x, y), y \in Y$, and by $\hat{F}$ we denote the map of $X$ into the set $C\langle Y, Z\rangle$, for which $\hat{F}(x)=F_{x}$ for every $x \in X$.

Also, if $G$ is a map from $X$ into $C\langle Y, Z\rangle$, then by $\tilde{G}$ we denote the map of $X \times Y$ into $Z$, for which $\tilde{G}(x, y)=G(x)(y)$ for every $(x, y) \in X \times Y$.

Hereafter, we assume the following. Let $\left(Y, k_{2}, \tau_{Y}^{n_{2}}\right)$ and $\left(Z, k_{3}, \tau_{Z}^{n_{3}}\right)$ be two generalized computer topological spaces and let $\mathcal{A}$ be the family of all generalized computer topological spaces $\left(X, k_{1}, \tau_{X}^{n_{1}}\right)$ such that the product space 
$\left(X \times Y, k, \tau_{X \times Y}^{n_{1}+n_{2}}\right)$ is well defined with an N-compatible $k$-adjacency. Consider the space $\left(C\langle Y, Z\rangle, k^{\prime}\right)$ defined with an $\mathrm{N}$-compatible $k^{\prime}$-adjacency as in Notation 1.

Definition 8. A topology $t$ on $\left(C\langle Y, Z\rangle, k^{\prime}\right)$ is called $\mathcal{A}$-splitting if for every $\left(X, k_{1}, \tau_{X}^{n_{1}}\right) \in \mathcal{A}$, GKD- $\left(k, k_{3}\right)$-continuity of a map $F: X \times Y \rightarrow Z$ implies $\operatorname{GKD}-\left(k_{1}, k^{\prime}\right)$-continuity of the map

$$
\hat{F}:\left(X, k_{1}, \tau_{X}^{n_{1}}\right) \rightarrow\left(C\langle Y, Z\rangle, k^{\prime}, t\right) .
$$

A topology $t$ on $\left(C\langle Y, Z\rangle, k^{\prime}\right)$ is called $\mathcal{A}$-admissible if for every space $(X$, $\left.k_{1}, \tau_{X}^{n_{1}}\right) \in \mathcal{A}$, GKD- $\left(k_{1}, k^{\prime}\right)$-continuity of a map

$$
G:\left(X, k_{1}, \tau_{X}^{n_{1}}\right) \rightarrow\left(C\langle Y, Z\rangle, k^{\prime}, t\right)
$$

implies GKD- $\left(k, k_{3}\right)$-continuity of the map

$$
\tilde{G}:\left(X \times Y, k, \tau_{X \times Y}^{n_{1}+n_{2}}\right) \rightarrow\left(Z, k_{3}, \tau_{Z}^{n_{3}}\right) .
$$

Theorem 4.2. Let $\left(Y, k_{2}, \tau_{Y}^{n_{2}}\right)$ and $\left(Z, k_{3}, \tau_{Z}^{n_{3}}\right)$ be two generalized computer topological spaces and let $\mathcal{A}$ be a family of all generalized computer topological spaces such that the product $Y \times Z$ is well defined with an $N$-compatible $k^{\prime}$ adjacency. Besides, let $\left(C\langle Y, Z\rangle, k^{\prime}\right)$ be the space defined with an $N$-compatible $k^{\prime}$-adjacency as in Notation 1. If a topology $t$ on $\left(C\langle Y, Z\rangle, k^{\prime}\right)$ is $\mathcal{A}$-splitting and $t^{\prime}$ is a topology such that $t^{\prime} \subset t$, then $t^{\prime}$ is also $\mathcal{A}$-splitting.

Proof. Let $\left(X, k_{1}, \tau_{X}^{n_{1}}\right) \in \mathcal{A}$ and let $F: X \times Y \rightarrow Z$ be a GKD- $\left(k, k_{3}\right)$ continuous map. Since the topology $t$ is an $\mathcal{A}$-splitting, the map

$$
\hat{F}:\left(X, k_{1}, \tau_{X}^{n_{1}}\right) \rightarrow\left(C\langle Y, Z\rangle, k^{\prime}, t\right)
$$

is GKD- $\left(k_{1}, k^{\prime}\right)$-continuous. Now, since for the topology $t^{\prime}$ satisfying $t^{\prime} \subset t$, the map

$$
i d:\left(C\langle Y, Z\rangle, k^{\prime}, t\right) \rightarrow\left(C\langle Y, Z\rangle, k^{\prime}, t^{\prime}\right)
$$

is also a GKD- $k^{\prime}$-continuous map. Thus, by Theorem 3.3, the map

$$
i d \circ \hat{F}:\left(X, k_{1}, \tau_{X}^{n_{1}}\right) \rightarrow\left(C\langle Y, Z\rangle, k^{\prime}, t^{\prime}\right)
$$

is also GKD- $\left(k_{1}, k^{\prime}\right)$-continuous and, therefore, the topology $t^{\prime}$ is $\mathcal{A}$-split ting.

Let us recall the following. If $t_{i}$ is topology on a set $X$, then in general the family $\cup\left\{t_{i}: i \in I\right\}$ need not be a topology on $X$. By the symbol $\vee\left\{t_{i}: i \in I\right\}$ we denote the topology on $X$ for which the family $\cup\left\{t_{i}: i \in I\right\}$ is a subbase.

Theorem 4.3. Let $\left(Y, k_{2}, \tau_{Y}^{n_{2}}\right)$ and $\left(Z, k_{3}, \tau_{Z}^{n_{3}}\right)$ be two generalized computer topological spaces and let $\mathcal{A}$ be the family of all generalized computer topological spaces $\left(X, k_{1}, \tau_{X}^{n_{1}}\right)$ such that the product $X \times Y$ is well defined with an $N$ compatible $k$-adjacency. On the space $\left(C\langle Y, Z\rangle, k^{\prime}\right)$ there exists the greatest $\mathcal{A}$-splitting topology denoted by $t_{g s}(\mathcal{A})$. Therefore,

$$
t_{\text {gs }}(\mathcal{A})=\vee\{t: t \quad \text { is } \mathcal{A} \text {-splitting }\} \text {. }
$$


Proof. We observe that for every $\mathcal{A}$-splitting topology $t$ we have that $t \subseteq t_{g s}(\mathcal{A})$. So, for the proof of this theorem it suffices to prove that the topology

$$
t_{\text {gs }}(\mathcal{A})=\vee\{t: t \text { is } \mathcal{A} \text {-splitting }\}
$$

is $\mathcal{A}$-splitting.

Let $\left(X, k_{1}, \tau_{X}^{n_{1}}\right) \in \mathcal{A}$ and let $F: X \times Y \rightarrow Z$ be a GKD- $\left(k, k_{3}\right)$-continuous map. We need to prove that the map

$$
\hat{F}:\left(X, k_{1}, \tau_{X}^{n_{1}}\right) \rightarrow\left(C\langle Y, Z\rangle, k^{\prime}, t_{g s}(\mathcal{A})\right)
$$

is $\operatorname{GKD}-\left(k_{1}, k^{\prime}\right)$-continuous.

Let $t$ be an $\mathcal{A}$-splitting topology. Then, $t \subseteq t_{g s}(\mathcal{A})$ and, therefore, the map

$$
\hat{F}:\left(X, k_{1}, \tau_{X}^{n_{1}}\right) \rightarrow\left(C\langle Y, Z\rangle, k^{\prime}, t\right)
$$

is a GKD- $\left(k_{1}, k^{\prime}\right)$-continuous map.

Thus, for every point $x_{0} \in X$ and for every $N_{k^{\prime}\left(\hat{F}\left(x_{0}\right), 1\right)} \subset C\langle Y, Z\rangle$, there is $N_{k_{1}}\left(x_{0}, 1\right) \subset X$ such that

$$
\hat{F}\left(N_{k_{1}}\left(x_{0}, 1\right)\right) \subset N_{k^{\prime}}\left(\hat{F}\left(x_{0}\right), 1\right) .
$$

So, it suffices to prove that the map

$$
\hat{F}:\left(X, k_{1}, \tau_{X}^{n_{1}}\right) \rightarrow\left(C\langle Y, Z\rangle, k^{\prime}, t_{g s}(\mathcal{A})\right)
$$

is topologically continuous. Let $U \in t_{g s}(\mathcal{A})$. Then, we obtain

$$
U=\bigcup\left\{U_{i_{1}} \cap \cdots \cap U_{i_{k(i)}}: i \in I\right\},
$$

where each set $U_{i_{m}}$ is an open set in the $\mathcal{A}$-splitting topology $t_{i_{m}}$. Since for every $i \in I$, each of topologies $t_{i_{1}}, \ldots, t_{i_{k(i)}}$ is $\mathcal{A}$-splitting, each map

$$
\hat{F}:\left(X, k_{1}, \tau_{X}^{n_{1}}\right) \rightarrow\left(C\langle Y, Z\rangle, k^{\prime}, t_{i_{m}}\right),
$$

is topologically continuous, where $t_{i_{m}} \in\left\{t_{i_{1}}, \ldots, t_{i_{k(i)}}\right\}$.

Thus, the sets $\hat{F}^{-1}\left(U_{i_{m}}\right) \in \tau_{X}^{n_{1}}$ and, therefore,

$$
\hat{F}^{-1}\left(U_{i_{1}} \cap \cdots \cap U_{i_{k(i)}}\right)=\hat{F}^{-1}\left(U_{i_{1}}\right) \cap \cdots \cap \hat{F}^{-1}\left(U_{i_{k(i)}}\right) \in \tau_{X}^{n_{1}} .
$$

Since

and

$$
U=\bigcup\left\{U_{i_{1}} \cap \cdots \cap U_{i_{k(i)}}: i \in I\right\}
$$

$$
\begin{aligned}
\hat{F}^{-1}(U) & =\hat{F}^{-1}\left(\bigcup\left\{U_{i_{1}} \cap \cdots \cap U_{i_{k(i)}}: i \in I\right\}\right) \\
& =\bigcup\left\{\hat{F}^{-1}\left(U_{i_{1}}\right) \cap \cdots \cap \hat{F}^{-1}\left(U_{i_{k(i)}}\right): i \in I\right\},
\end{aligned}
$$

we obtain

$$
\hat{F}^{-1}(U) \in \tau_{X}^{n_{1}}
$$

Thus the map

$$
\hat{F}:\left(X, k_{1}, \tau_{X}^{n_{1}}\right) \rightarrow\left(C\langle Y, Z\rangle, k^{\prime}, t_{g s}(\mathcal{A})\right)
$$

is topologically continuous, which completes the proof. 
Theorem 4.4. Let $\left(Y, k_{2}, \tau_{Y}^{n_{2}}\right)$ and $\left(Z, k_{3}, \tau_{Z}^{n_{3}}\right)$ be two generalized computer topological spaces and let $\mathcal{A}$ be the family of all generalized computer topological spaces $\left(X, k_{1}, \tau_{X}^{n_{1}}\right)$ such that the product $X \times Y$ is well defined with an $N$ compatible $k$-adjacency. If a topology $t$ on $\left(C\langle Y, Z\rangle, k^{\prime}\right)$ is $\mathcal{A}$-admissible and $t^{\prime}$ is a topology such that $t \subset t^{\prime}$, then $t^{\prime}$ is also $\mathcal{A}$-admissible.

Proof. Let $\left(X, k_{1}, \tau_{X}^{n_{1}}\right) \in \mathcal{A}$ and let

$$
G:\left(X, k_{1}, \tau_{X}^{n_{1}}\right) \rightarrow\left(C\langle Y, Z\rangle, k^{\prime}, t^{\prime}\right)
$$

be $\operatorname{GKD}-\left(k, k_{3}\right)$-continuous. We must prove that the map

$$
\tilde{G}:\left(X \times Y, k, \tau_{X \times Y}^{n_{1}+n_{2}}\right) \rightarrow\left(Z, k_{3}, \tau_{Z}^{n_{3}}\right)
$$

is $\operatorname{GKD}-\left(k, k_{3}\right)$-continuous.

Indeed, since $t \subseteq t^{\prime}$, the map

$$
G:\left(X, \tau_{X}^{n_{1}}\right) \rightarrow(C\langle Y, Z\rangle, t)
$$

is topologically continuous. Also, since the map $G:\left(X, k_{1}, \tau_{X}^{n_{1}}\right) \rightarrow(C\langle Y, Z\rangle$, $\left.k^{\prime}, t^{\prime}\right)$ is $\operatorname{GKD}-\left(k, k_{3}\right)$-continuous, the map

$$
G:\left(X, k_{1}, \tau_{X}^{n_{1}}\right) \rightarrow\left(C\langle Y, Z\rangle, k^{\prime}, t\right)
$$

is also GKD- $\left(k_{1}, k^{\prime}\right)$-continuous.

Now, since the topology $t$ is $\mathcal{A}$-admissible, the map

$$
\tilde{G}:\left(X \times Y, k, \tau_{X \times Y}^{n_{1}+n_{2}}\right) \rightarrow\left(Z, k_{3}, \tau_{Z}^{n_{3}}\right)
$$

is $\operatorname{GKD}-\left(k, k_{3}\right)$-continuous. By this fact we have that the topology $t^{\prime}$ is $\mathcal{A}$ admissible.

Theorem 4.5. Let $\left(Y, k_{2}, \tau_{Y}^{n_{2}}\right)$ and $\left(Z, k_{3}, \tau_{Z}^{n_{3}}\right)$ be two generalized computer topological spaces and let $\mathcal{A}$ be the family of all generalized computer topological spaces $\left(X, k_{1}, \tau_{X}^{n_{1}}\right)$ such that the product $X \times Y$ is well defined with an $N$ compatible $k$-adjacency. If a topology $t$ is $\mathcal{A}$-splitting and $t^{\prime}$ is an $\mathcal{A}$-admissible topology on $\left(C\langle Y, Z\rangle, k^{\prime}\right)$ such that $\left(C\langle Y, Z\rangle, k^{\prime}, t^{\prime}\right) \in \mathcal{A}$, then $t \subseteq t^{\prime}$.

Proof. We observe that the map

$$
G:=1_{C\langle Y, Z\rangle}:\left(C\langle Y, Z\rangle, k^{\prime}, t^{\prime}\right) \rightarrow\left(C\langle Y, Z\rangle, k^{\prime}, t^{\prime}\right)
$$

is GKD- $k^{\prime}$-continuous.

Also, since the topology $\left(C\langle Y, Z\rangle, k^{\prime}, t^{\prime}\right) \in \mathcal{A}$, we have that the product $C\langle Y, Z\rangle \times Y$ is well defined with an $N$-compatible $k$-adjacency. Now, since the topology $t^{\prime}$ is $\mathcal{A}$-admissible, the map

$$
\tilde{G} \equiv F:\left(C\langle Y, Z\rangle, k^{\prime}, t^{\prime}\right) \times\left(Y, k_{2}, \tau_{Y}^{n_{2}}\right) \rightarrow\left(Z, k_{3}, \tau_{Z}^{n_{3}}\right)
$$

is $\operatorname{GKD}-\left(k, k_{3}\right)$-continuous.

Finally, since the topology $t$ is $\mathcal{A}$-splitting, the map

$$
\hat{F}:\left(C\langle Y, Z\rangle, k^{\prime}, t^{\prime}\right) \rightarrow\left(C\langle Y, Z\rangle, k^{\prime}, t\right)
$$


is GKD- $k^{\prime}$-continuous. By this fact we have that the map

$$
\hat{F}:\left(C\langle Y, Z\rangle, t^{\prime}\right) \rightarrow(C\langle Y, Z\rangle, t)
$$

is topologically continuous. Also, we observe that $\hat{F}=i d$. Thus, $t \subseteq t^{\prime}$.

\section{Concluding remarks}

We give and study the notion of GKD- $\left(k_{0}, k_{1}\right)$-continuous function between (generalized) computer topological spaces with $k_{i}$-adjacency, $i \in\{0,1\}$. This study is an application on function spaces. Let us now adopt a forgetful functor from $K D T C$ into $D T C$, denoted by

$$
F^{*}: K D T C \rightarrow D T C .
$$

Then, the forgetful functor $F^{*}$ transforms a computer topological space $X_{n, k}$ into a discrete topological space (or digital image) with $k$-adjacency $(X, k)$ $[5,6,9,10,13]$. Furthermore, the current $\mathrm{KD}-\left(k_{0}, k_{1}\right)$-continuity is also transformed into the digital $\left(k_{0}, k_{1}\right)$-continuity in $D T C$. Thus we can study various properties of a digital function space in $D T C$.

\section{References}

[1] C. Berge, Graphs and Hypergraphs, 2nd ed., North-Holland, Amsterdam, 1976.

[2] L. Boxer, A classical construction for the digital fundamental group, J. Math. Imaging Vision 10 (1999), no. 1, 51-62.

[3] J. Dontchev and H. Maki, Groups of $\theta$-generalized homeomorphisms and the digital line, Topology Appl. 95 (1999), no. 2, 113-128.

[4] G. Gierz, K. H. Hofmann, K. Keimel, J. D. Lawson, M. Mislove, and D. S. Scott, A Compendium of Continuous Lattices, Springer-Verlag, Berlin-New York, 1980.

[5] S. E. Han, Computer topology and its applications, Honam Math. J. 25 (2003), no. 1, $153-162$.

[6] _ Algorithm for discriminating digital images w.r.t. a digital $\left(k_{0}, k_{1}\right)$-homeomorphism, J. Appl. Math. Comput. 18 (2005), no. 1-2, 505-512.

[7] _ Non-product property of the digital fundamental group, Inform. Sci. 171 (2005), no. 1-3, 73-91.

[8] _ On the simplicial complex stemmed from a digital graph, Honam Math. J. 27 (2005), no. 1, 115-129.

[9] Connected sum of digital closed surfaces, Inform. Sci. 176 (2006), no. 3, 332348.

[10] - Digital fundamental group and Euler characteristic of a connected sum of digital closed surfaces, Inform. Sci. 177 (2007), no. 16, 3314-3326.

[11] _ Strong $k$-deformation retract and its applications, J. Korean Math. Soc. 44 (2007), no. 6, 1479-1503.

[12] Continuities and homeomorphisms in computer topology and their applications, J. Korean Math. Soc. 45 (2008), no. 4, 923-952.

[13] _ Equivalent $\left(k_{0}, k_{1}\right)$-covering and generalized digital lifting, Inform. Sci. 178 (2008), no. 2, 550-561.

[14] _ The k-homotopic thinning and a torus-like digital image in $\mathbb{Z}^{n}$, J. Math. Imaging Vision 31 (2008), no. 1, 1-16.

[15] E. Khalimsky, R. Kopperman, and P. R. Meyer, Computer graphics and connected topologies on finite ordered sets, Topology Appl. 36 (1990), no. 1, 1-17. 
[16] I. S. Kim, S. E. Han, and C. J. Yoo, The pasting property of digital continuity, Acta Applicandae Mathematicae (2009), doi 10.1007/s 10440-008-9422-0, Online first publication.

[17] T. Y. Kong and A. Rosenfeld, Topological Algorithms for the Digital Image Processing, Elsevier Science, Amsterdam, 1996.

[18] E. Melin, Extension of continuous functions in digital spaces with the Khalimsky topology, Topology Appl. 153 (2005), no. 1, 52-65.

[19] T. Noiri, On $\delta$-continuous functions, J. Korean Math. Soc. 16 (1979/80), no. 2, 161-166.

[20] A. Rosenfeld, Arcs and curves in digital pictures, J. Assoc. Comput. Mach. 20 (1973), 81-87.

[21] J. Šlapal, Digital Jordan curves, Topology Appl. 153 (2006), no. 17, 3255-3264.

SANG-EON HAN

FACULTy of Liberal EdUCATion,

Institute of Pure and Applied Mathematics

Chonbuk National University

JEONBuK 561-756, KoreA

E-mail address: sehan@chonbuk.ac.kr

D. N. Georgiou

University of Patras

Department of Mathematics

26500 Patras, Greece

E-mail address: georgiou@math.upatras.gr 